\title{
Propagation and Transmission of Alfvén Waves in Rotating Magnetars
}

\author{
Yasufumi Kojima and Taishi Okita \\ Department of Physics, Hiroshima University, Higashi-Hiroshima 739-8526 Japan \\ kojima@theo.phys.sci.hiroshima-u.ac.jp
}

\begin{abstract}
We study the propagation and transmission of Alfvén waves in the context of cylindrical geometry. This approximates the polar cap region of aligned pulsar with strong magnetic fields. Non-propagating region appears in the presence of rotation. The displacement current further prevents the low frequency modes from propagating near the stellar surface. The transmission rates to the exterior through the surface are calculated. The rates increase with the frequency and the magnetic field strength. The transmission also depends on the helicity states of the waves, but the difference becomes small in the high frequency regime. We also point out the possibility of the spin-up by outgoing wave emission in the low frequency regime, if a certain condition holds.
\end{abstract}

Subject headings: Star: Magnetic Fields - Star: Neutron-Star: Magnetar-Gamma Rays: Bursts

\section{Introduction}

Soft Gamma Repeaters (SGRs) and Anomalous X-ray Pulsars (AXPs) belong to a rare class among thousands of neutron stars. Both objects are different from so far known neutron stars in their radiation spectrum and spin periods. SGR-like outbursts were also discovered in AXPs (Gavriil, Kaspi \& Woods 2002; Kaspi et al. 2003), and hence there are similarities between two peculiar neutron stars. They are likely to be young and isolated, but have intense magnetic fields in the $10^{14}-10^{15} \mathrm{G}$ range. See Mereghetti \& Stella (1995); Kouveliotou et al. (1998) for their initial observations and also Woods (2003) for recent reviews. Further evidence of the strong magnetic fields has recently come from spectral line feature(Ibrahim et al. 2003). These objects are called as magnetars (Thompson \& Duncan 1993, 1995, 1996). The magnetar model has been able to explain the peculiar observational properties. See e.g. Thompson (2000); Lyutikov et al. (2002) as recent reviews. The burst emission and non-thermal X-ray radiation are supplied by the decay of strong magnetic fields. Twisted magnetic fields relevant to the activities are transported from the core to the surface by ambipolar diffusion in the magnetars model (Thompson et al. 2002).

The Alfvén waves are likely to be excited by sudden disturbances in various magnetized objects. Short duration bursts in SGRs may be associated with starquakes driven by magnetic stress, since 
the similarity in statistical property is reported between the SGRs and earthquakes (Cheng et al. 1996; Gogus et al. 1999, 2000). The Alfvén waves are very interesting in their property and physical mechanism. The fluid displacement is perpendicular to both of the wave vector and the magnetic field, and restoring force arises from the magnetic tension of the field lines. The plane Alfvén waves of finite amplitude can propagate at constant speed in a homogeneous incompressible medium without any distortion of the waveform. This contrasts with sound waves, which may steepen to form shocks because of the non-linearity. As for the physical mechanism, torsional Alfvén waves can transport angular momentum, and account for the spindown of rotating objects, such as magnetized stars with convective envelopes, interstellar clouds threaded by the Galactic magnetic fields and so on. See e.g. Shu (1992) for details.

We consider the Alfvén waves produced by shaking the magnetic field lines in a rotating magnetar in this paper. The origin of abrupt disturbances relevant to the bursts is not addressed here, but the subsequent propagation and ejection to the exterior are examined. The total energy in shorter duration bursts is $\Delta E<10^{41} \mathrm{ergs}$, and is a small fraction of available magnetic energy $\sim 10^{-5} E_{B}$, where the total magnetic energy $E_{B}$ is estimated by the expected dipole field strength $B=10^{14}-10^{15} \mathrm{G}$. On the other hand, giant flares with $\Delta E \sim 10^{44}$ ergs should involve more drastic change of magnetic field configuration on a global scale (Ioka 2001; Woods et al. 2001). We here use the linearized perturbation equations, which are applicable to less energetic events $\Delta E / E_{B} \propto(\delta B / B)^{2} \ll 1$, in the shorter duration bursts. Our results of the wave propagation may not be applied to the giant flares, but there may be some similarities in nature even for $\delta B \sim B$. The ejection of Alfvén waves to the exterior was already estimated so far, but our model is beyond a simple model. We here explicitly calculate the transmission in a cylindrical geometry. We also calculate the energy and angular momentum extracted by the waves through the stellar surface. In section 2, we provide a geometrically simplified model. The propagation in the neutron-star crust and transmission to the exterior can be examined in a concrete way owing to the simplified model. The shear in the solid crust acts as additional restoring force. Therefore, the shear-Alfvén waves may be more adequate for the name. The transmission rate is numerically calculated for the waves with higher frequency. We discuss the implication of our results in section 3.

\section{Alfvén Waves in Rotating Cylinder}

\subsection{Model}

In this paper, we study the propagation of the shear-Alfvén waves through a neutron-star crust and the transmission to the exterior. The star is assumed to rotate around $z$-axis with a constant angular velocity $\Omega$. The magnetic field is uniform along the $z$-axis, i.e. $\vec{B}=B_{0} \vec{e}_{z}$, where $B_{0}$ is a constant. Our consideration is limited to a cylindrically symmetric slab region within the radius $\varpi_{*}$. The magnetic fields are rather easily incorporated in the cylindrical model. Carroll et al. (1986) studied the oscillation spectra of the magnetized/unmagnetized cylindrical stars. Their 
results show that the periods of the torsional and shear modes are in good agreement with those of spherically symmetric unmagnetized star. This suggests that the approximation is good for some modes.

The cylindrical approximation may be adequate for the polar cap region and the interior for an aligned rotator. In this case, the radius $\varpi_{*}$ is given by the size of the polar cap, $\varpi_{*} \leq R \sin \theta_{p}=$ $\sqrt{R^{3} \Omega / c} \approx 10^{4}(T / 1 \mathrm{~s})^{-1 / 2} \mathrm{~cm}$, where $R \approx 10^{6} \mathrm{~cm}$ is stellar radius and $T$ is the rotational period. The exterior magnetic field lines originated from this region are extended to infinity. On the other hand, the field lines outside the polar cap become closed ones. The plasma along the closed lines corotates with the central star. In our cylindrical model, we consider the shaking of open field lines only, which may lead to particle acceleration, magnetic reconnection, wind, and eventually to the radiation. Explicit treatment of the energy transfer to the radiation is also beyond the scope of this paper.

The shear stress in the crust also acts as a restoring force, and is coupled to the Alfvén waves. The solid crust ranges from $z=-d$ to the surface $z=0$. The depth $d$ of the outer crust is given by $d \approx 10^{5} \mathrm{~cm}$. Our consideration is limited to thin outer crust only. The slab geometry is valid there because $d \ll R$. Gravitational acceleration in this region is almost constant and is approximated as $-g \vec{e}_{z}$, with $g=10^{14} \mathrm{~cm} \mathrm{~s}^{-2}$. The density distribution $\rho(z)$ is given by the integration of hydrostatic equation for neutron star crust $(|z|<d)$, where degenerate electron pressure is dominated. The analytic expression is available as (Blaes et al. 1989)

$$
\rho=8.0 \times 10^{1}\left[(|z| / \mathrm{cm})+2.5 \times 10^{-4}(|z| / \mathrm{cm})^{2}\right]^{3 / 2} \mathrm{~g} \mathrm{~cm}^{-3} .
$$

This explicit form will be used for the numerical calculations in the subsequent sections.

\subsection{Wave equation}

We consider the propagation of shear-Alfvén waves based on the linearized perturbation theory. In the deep interior, gravitational force is so large that the displacements to the vertical direction are not easily to be induced. The horizontal displacements of the disturbances are likely to be dominated, and they are coupled with the Alfvén waves. Some restrictions are imposed on the displacement vector $\vec{\xi}$ of the materials in order to extract the waves coupled with shear and magnetic stress. The waves propagating along the $z$-axis are assumed to satisfy $\xi_{z}=\vec{\nabla} \cdot \vec{\xi}=0$. This means that the waves are transverse and are decoupled from the compressional modes. The Lagrangian perturbations of density and pressure are zero because they are proportional to $\vec{\nabla} \cdot \vec{\xi}$, i.e. $\Delta \rho / \rho=$ $-\vec{\nabla} \cdot \vec{\xi}=0, \Delta p / p=-(\partial \log p / \partial \log \rho)_{a d} \vec{\nabla} \cdot \vec{\xi}=0$.

The general forms of the displacements can be expressed by the Fourier and Bessel functions with respect to time and cylindrical radius, respectively. We further simplify the displacements by assuming nodeless functions in cylindrically radial direction, i.e. neglecting the radial structure. 
The regular form near the $z$-axis is simply given in the cylindrical coordinate $(\varpi, \phi, z)$ as

$$
\vec{\xi} \propto\left(\vec{e}_{\varpi} \pm i \vec{e}_{\phi}\right) \varpi^{m-1} \xi_{ \pm m \omega}(z) e^{-i(\omega t \mp m \phi)},
$$

where $m$ is a positive integer, $m \geq 1$. The mode for the upper sign in eq.(2) is said to have positive helicity, whereas that for lower sign has negative helicity. We may limit the Fourier mode to a certain frequency region, using a general relation $\xi_{ \pm m-\omega}=\xi_{\mp}^{*}{ }_{m} \omega$, where ${ }^{*}$ means complex conjugate. We define the frequency in corotating frame $\sigma_{ \pm} \equiv \omega \mp m \Omega$ for each helicity state $\xi_{ \pm}$ and limit the range to $\sigma_{ \pm} \geq 0$. From now on, we will omit the suffixes $m \omega$ in order to avoid complicated notations. The displacement $(2)$ satisfies $(\vec{\nabla} \times \vec{\xi})_{z}=0$ and is therefore decoupled from the vorticity as it is desired. The displacement corresponding to $m=1$ has clear meaning, and can be written in the Cartesian coordinates as $\vec{\xi}=\left(\vec{e}_{x} \pm i \vec{e}_{y}\right) \xi_{ \pm} e^{-i \omega t}$. This mode represents uniform motion in horizontal direction. A slightly different treatment is necessary for the axially symmetric perturbation $m=0$. The regular displacement satisfying above conditions is given as $\vec{\xi}=\xi_{\phi}(t, \varpi) \varpi \vec{e}_{\phi}$, which corresponds to the velocity perturbation $\delta \vec{v}=\left(\partial \xi_{\phi} / \partial t\right) \varpi \vec{e}_{\phi} \equiv \delta \Omega \varpi \vec{e}_{\phi}$. This kind of perturbation merely represents impulsive jump of angular velocity as $\Omega \rightarrow \Omega+\delta \Omega$, and is no longer considered in this paper.

The restoring forces for the modes are elastic shear stress $\delta S_{i}$ and electromagnetic force $\delta F_{i}$. The linearized equation of motion can be written as

$$
\rho\left(\frac{\partial}{\partial t} \delta v_{i}+(\vec{v} \cdot \vec{\nabla}) \delta v_{i}+(\delta \vec{v} \cdot \vec{\nabla}) v_{i}\right)=\delta S_{i}+\delta F_{i}
$$

where $\vec{v}=\varpi \Omega \vec{e}_{\phi}$, and the relation between the displacement and velocity perturbation is

$$
\delta v_{i}=\frac{\partial}{\partial t} \xi_{i}+(\vec{v} \cdot \vec{\nabla}) \xi_{i}-(\vec{\xi} \cdot \vec{\nabla}) v_{i}
$$

The shear stress $\delta S_{i}$ associated with deformation is

$$
\delta S_{i}=\nabla_{j}\left[\mu\left(\frac{\partial \xi^{i}}{\partial x^{j}}+\frac{\partial \xi^{j}}{\partial x^{i}}\right)\right],
$$

with shear modulus $\mu=4.8 \times 10^{27}\left(\rho / 10^{11} \mathrm{~g} \mathrm{~cm}^{-3}\right)^{4 / 3} \mathrm{erg} \mathrm{cm}^{-3}$ (Baym, \& Pines 1971). The electromagnetic force is given by

$$
\delta \vec{F}=\delta \rho_{e} \vec{E}+\rho_{e} \delta \vec{E}+\frac{1}{c}(\delta \vec{j} \times \vec{B}+\vec{j} \times \delta \vec{B}) .
$$

The crust is a perfect conductor, so that the electric fields for both unperturbed and perturbed states are induced by the material motion as

$$
\vec{E}=-\frac{1}{c}(\vec{v} \times \vec{B})=-\frac{\varpi \Omega B_{0}}{c} \vec{e}_{\varpi}
$$




$$
\delta \vec{E}=-\frac{1}{c}(\delta \vec{v} \times \vec{B}+\vec{v} \times \delta \vec{B}) .
$$

The electric charge and current for the unperturbed state are

$$
\rho_{e}=-\frac{1}{2 \pi c} \Omega B_{0}, \quad \vec{j}=\rho_{e} \vec{v}
$$

These quantities (7) and (9) satisfy the force balance $\rho_{e} \vec{E}+\vec{j} \times \vec{B} / c=0$. These expressions are valid in the region smaller than light cylinder $c / \Omega$ or actual stellar radius $R$. Otherwise, we would need better approximation beyond the uniformly rotating cylindrical model. Therefore, our present model may be adequate to the behavior near the $z$-axis. As will be confirmed by the explicit forms of $\vec{E}$ and $\delta \vec{E}$, the first term $\delta \rho_{e} \vec{E}(=(\vec{\nabla} \cdot \delta \vec{E} / 4 \pi) \vec{E})$ in eq. $(6)$ is proportional to $(\Omega \varpi / c)^{2}$ and is smaller than other terms near the $z$-axis, i.e. for the region $\varpi^{2}<\xi / \xi^{\prime \prime}$. We therefore neglect the first term $\delta \rho_{e} \vec{E}$ in the propagation of Alfvén waves. Eliminating $\delta \vec{E}$ by eq.(8), the Lorentz force (6) is reduced to

$$
\delta \vec{F}=\frac{1}{c}\left(\delta \vec{j}-\rho_{e} \delta \vec{v}\right) \times \vec{B} .
$$

The perturbation of electric current is determined by the Maxwell's equations:

$$
\begin{aligned}
& \nabla \times \delta \vec{E}=-\frac{1}{c} \frac{\partial}{\partial t} \delta \vec{B} \\
& \nabla \times \delta \vec{B}=\frac{4 \pi}{c} \delta \vec{j}+\frac{1}{c} \frac{\partial}{\partial t} \delta \vec{E} .
\end{aligned}
$$

From eqs.(8) and (11), the perturbations of electro-magnetic fields are expressed in terms of the displacement vector as

$$
\begin{aligned}
& \delta \vec{B}=B_{0} \nabla_{z} \vec{\xi}=B_{0} \varpi^{m-1} \frac{d \xi_{ \pm}}{d z}\left(\vec{e}_{\varpi} \pm i \vec{e}_{\phi}\right) e^{-i(\omega t \mp m \phi)} \\
& \delta \vec{E}=\frac{B_{0} \varpi^{m-1}}{c}\left[\mp(\omega \mp m \Omega) \xi_{ \pm}\left(\vec{e}_{\varpi} \pm i \vec{e}_{\phi}\right)+\Omega \varpi \frac{d \xi_{ \pm}}{d z} \vec{e}_{z}\right] e^{-i(\omega t \mp m \phi)} .
\end{aligned}
$$

The stellar rotation induces the $z$-component of the perturbed electric field, which is important for angular momentum transfer as discussed in section 2.6. Using these expressions and eliminating $\delta \vec{j}$ by eq.(12), eq.(3) is eventually reduced to

$$
\frac{d}{d z}\left(\mu+\frac{B_{0}^{2}}{4 \pi}\right) \frac{d}{d z} \xi_{ \pm}+V_{ \pm} \xi_{ \pm}=0
$$


where

$$
\begin{aligned}
& V_{ \pm}=\left(\rho+\frac{B_{0}^{2}}{4 \pi c^{2}}\right)(\omega \mp m \Omega)[\omega \mp(m h-2) \Omega], \\
& h=\frac{4 \pi c^{2} \rho}{4 \pi c^{2} \rho+B_{0}^{2}} .
\end{aligned}
$$

\subsection{Propagation}

The propagation of Alfvén waves is studied in different contents. In a certain limit of parameters, our basic equation (15) should be reduced to one previously studied. In the limit of non-rotating case, eq.(15) is reduced to one found by Blaes et al. (1989). The term $V_{ \pm}$is independent of the helicity state and is positive definite in this case. Another interesting limit of eq.(15) is obtained when both shear and relativistic effects are neglected. This limit corresponds to $\mu \rightarrow 0$ and $c^{2} \rightarrow \infty$. In this case, the term $V_{ \pm}$becomes negative for a certain frequency region. Hence, the modes are non-propagating (evanescent). See e.g. Chandrasekhar (1961). We will discuss the evanescent property below.

We consider the short-wavelength limit in order to see whether or not the waves propagate. The local dispersion relation is obtained by setting $\xi_{ \pm} \propto e^{i k z}$ in eq.(15)

$$
-v^{2} k^{2}+(\omega \mp m \Omega)(\omega \mp(m h-2) \Omega)=0,
$$

where $v$ is the velocity defined as

$$
v=c\left(\frac{4 \pi \mu+B_{0}^{2}}{4 \pi \rho c^{2}+B_{0}^{2}}\right)^{1 / 2}
$$

In the limit of $\mu \rightarrow 0$ and $c^{2} \rightarrow \infty$, the classical Alfvén wave velocity is recovered, i.e. $v=$ $B_{0} / \sqrt{4 \pi \rho}$. The shear acts as the restoring force deep in the crust unless $B_{0} \gg 2 \times 10^{14} \mathrm{G}$. The displacement current contributes to additional inertia and is always important near the surface, where the propagation velocity becomes $c$. Solving eq.(18) for the frequencies $\sigma_{ \pm} \equiv \omega \mp m \Omega \geq 0$, we have

$$
\sigma_{ \pm}=\mp\left[1+\frac{m}{2}(1-h)\right] \Omega+\left\{\left[1+\frac{m}{2}(1-h)\right]^{2} \Omega^{2}+v^{2} k^{2}\right\}^{1 / 2}
$$

We do not have to consider the modes with negative frequency $\sigma_{ \pm}<0$ because of the correspondence $\left(\xi_{ \pm}, \sigma\right) \leftrightarrow\left(\xi_{\mp}^{*},-\sigma\right)$ as mentioned in section 2.2. The difference between two helicity modes is clear in weak magnetic field limit, i.e. $v k / \Omega \rightarrow 0$ and $h \rightarrow 1$. In this limit, the frequencies in the 
corotating frame are given by $\sigma_{-} \approx 2 \Omega$ and $\sigma_{+} \approx v^{2} k^{2} /(2 \Omega)$. The mode $\xi_{-}$with frequency $\sigma_{-}$ represents an inertial wave due to Coriolis force. On the other hand, the mode $\xi_{+}$with smaller frequency $\sigma_{+}\left(\ll \sigma_{-}\right)$represents a drift wave. Our problem is limited to cylindrical geometry, but the similar oscillations are also possible in spherical geometry. See e.g. Levin \& D'Angelo (2004) for comparison in the non-relativistic limit, although careful treatment is necessary to convert results between different geometry.

Like the Alfvén waves in classical treatment, the waves with low frequencies become nonpropagating due to the Coriolis force. The mode $\xi_{+}$is always propagating as far as $\sigma_{+} \geq 0$, whereas the mode $\xi_{-}$is non-propagating for the frequency region $0 \leq \sigma_{-} / \Omega \leq 2+m(1-h)$. The condition becomes $0 \leq \sigma_{-} / \Omega \leq 2$, when the relativistic effects are neglected. The evanescent property in our problem depends on the spatial position through $h(z)$, which is calculated for the density distribution (1) and constant magnetic field strength $B_{0}$. It is general that the function $h$ decreases from $h \sim 1$ in deep interior to $h \sim 0$ near the surface with low density. The evanescent region therefore prevails near the surface. In Fig.1, we demonstrate the location of evanescent region using the local dispersion relation. The critical frequency $\sigma_{-} / \Omega=2+m(1-h)$ is shown. The depth of the evanescent region increases with azimuthal wave number $m$ as well as the magnetic field strength $B_{0}$.

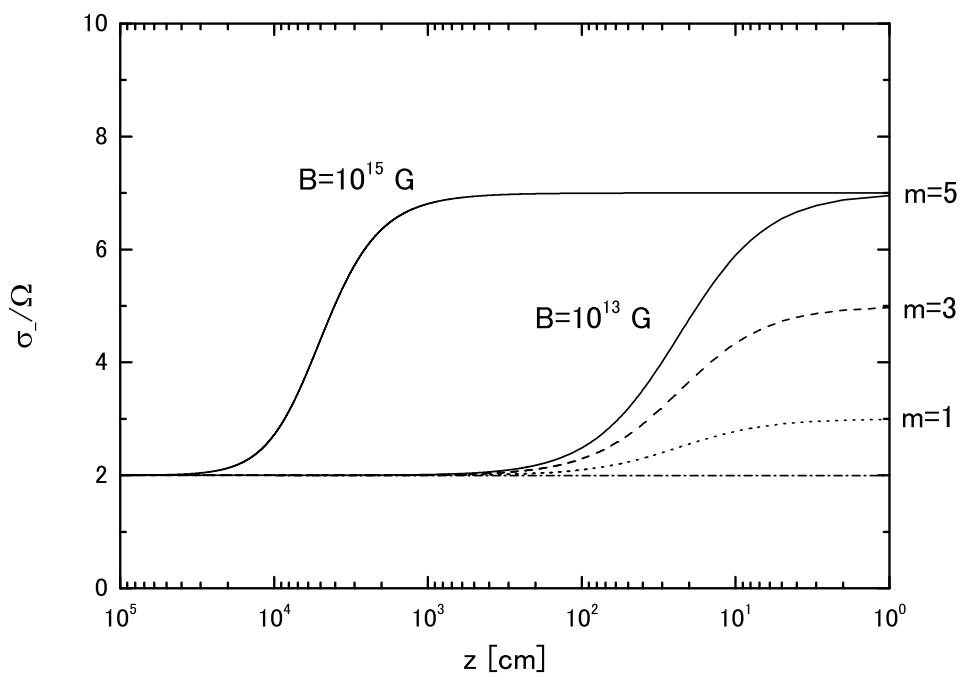

Fig. 1. - The critical angular frequency $\sigma_{-} / \Omega=2+m(1-h)$ as a function of the depth from the surface. Evanescent region for negative helicity mode $\xi_{-}$is given by the condition $0<\sigma_{-} / \Omega<2+m(1-h)$. The critical frequencies are shown for $m=1,3,5$ with $B_{0}=10^{13} \mathrm{G}$ and $m=5$ with $B_{0}=10^{15} \mathrm{G}$. Note that the evanescent condition becomes $0<\sigma_{-} / \Omega<2$ denoted by a dash-dotted line, if all relativistic effects are neglected. The evanescent region near the surface enlarges with increase of $m$ and the magnetic field strength. 
The phase and group velocities of the shear-Alfvén waves in the inertial frame are respectively given by $v_{p}=\omega / k$ and $v_{g}=\partial \omega / \partial k$. The following relation is easily calculated

$$
\frac{v_{p} v_{g}}{v^{2}}=\frac{\omega}{\omega \pm \frac{1}{2}[2-m(1+h)] \Omega}=\frac{\sigma_{ \pm} \pm m \Omega}{\sigma_{ \pm} \pm \frac{1}{2}[2+m(1-h)] \Omega}
$$

This shows an interesting property that the phase and group velocities of the mode $\xi_{-}$are opposite in the propagating direction for the frequency range $[2+m(1-h)] \Omega<\sigma_{-}<m \Omega$, which is possible for $m \geq 3$. The lower bound comes from the condition for the wave propagation.

In Fig.2, we show $1 / N^{2}=\omega^{2} /\left(c^{2} k^{2}\right)$ as a function of $\sigma_{-} / \Omega=\omega / \Omega+m$, using the local dispersion relation (18). The frequency corresponding to negative value of $1 / N^{2}$ mean evanescent. The non-propagating frequency is located in low frequency region. We now consider the propagation of the negative helicity mode with $2<\sigma_{-} / \Omega<m$. The mode propagates as a wave in deep interior, and may leak out through to the exterior. The amplitude of the outgoing wave becomes small through the evanescent region. It is important to know the magnitude. The damping rate depends on the frequency and the depth $d_{*}$. The exponential damping factor $e^{-\kappa d_{*}}$ is estimated as $\kappa d_{*} \approx|N| \omega d_{*} / c$, which is not so large as roughly estimated $\sim 10^{-4}$ for large $m$. This means that the damping of the amplitude is not so significant, and therefore the disturbances of some modes are penetrated into the surface.

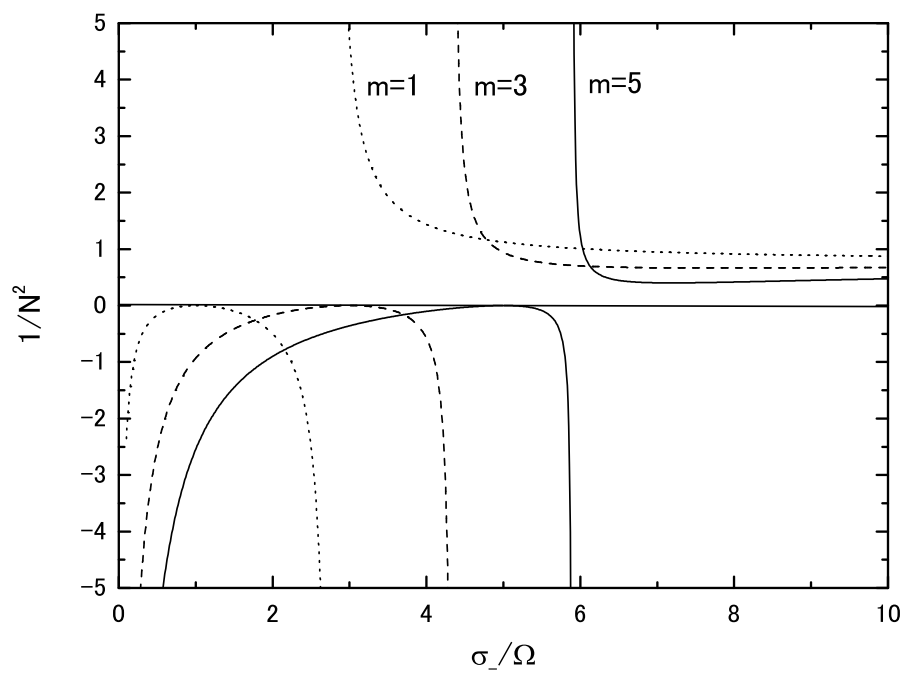

Fig. 2.- Dispersion relation of negative helicity mode $\xi_{-}$as a function of angular frequency $\sigma_{-} / \Omega$ at $z=10 \mathrm{~cm}$ from the surface. 


\subsection{Exterior}

There are at least two possibilities as for the exterior of the stars. One is that surrounding plasma outside the star corotates with the same angular velocity. When the plasma is frozen in closed magnetic field lines, such a corotation is realized. However, the plasma freely moves to infinity along open field lines, so that the angular velocity is arbitrary in this case. We also consider this situation by assuming that the plasma exterior to the star is static in the inertial frame. The difference of the plasma motion in the background state is not so important for the high frequency modes of the perturbations $(\omega \gg \Omega)$, but crucial for the low frequency modes, as discussed below.

When the plasma corotates with the same angular velocity $\Omega$, the perturbation equation corresponds to the limit of eq.(15) with $\rho=\mu=0$. The wave solution is possible for the modes with $(\omega \mp m \Omega)(\omega \pm 2 \Omega)>0$ and outgoing wave is expressed as

$$
\xi_{ \pm}=A_{ \pm} \exp \left[i k_{ \pm} z\right]
$$

where

$$
k_{ \pm}=\frac{[(\omega \mp m \Omega)(\omega \pm 2 \Omega)]^{1 / 2}}{c} .
$$

We assume that the interior and exterior solutions are continuously matched, so that the boundary condition of the displacement at $z=0$ can be expressed as

$$
\frac{d \xi_{ \pm}}{d z}=i k_{ \pm} \xi_{ \pm}
$$

We next consider the non-rotating plasma in the exterior of the star. In this case, outgoing boundary condition at the surface is replaced as

$$
\frac{d \xi_{ \pm}}{d z}=i \frac{\omega}{c} \xi_{ \pm}
$$

The shaking of the magnetic field lines induces electromagnetic radiation in the exterior vacuum. Since $\delta B_{z}=0$ everywhere, the perturbation should be matched with the transverse magnetic mode with the cylindrical wave guide (Jackson 1975). The perpendicular components of elec-

tric fields, i.e. $\delta E_{\varpi}, \delta E_{\phi}$ are continuous. They are expressed by $\vec{\xi}$ in the interior and vary as $\propto \exp \{i \omega(z / c-t)\}$ in the exterior. Thus we have the boundary condition (25) at the surface.

\subsection{Transmission coefficient}

In this section, we consider how much Alfvén waves excited in the crust are transmitted to the exterior. We numerically solve eq.(15) from the deep interior $z \approx 10^{5} \mathrm{~cm}$, corresponding to 
the neutron drip in the density, to the surface. The following asymptotic solution is obtained in the deep crust (Blaes et al. 1989). It is a sum of outgoing and reflecting waves, which respectively propagate to $+z$ and $-z$ directions. We normalize the amplitude of outgoing wave to unity for simplicity and denote that of reflecting wave by $R_{ \pm}$.

$$
\xi_{ \pm} \approx|z|^{-\frac{7}{4}}\left\{\exp \left[i \int \frac{\Gamma_{ \pm}}{v} d z\right]+R_{ \pm} \exp \left[-i \int \frac{\Gamma_{ \pm}}{v} d z\right]\right\}
$$

Here, $\Gamma_{ \pm}$is an effective frequency of each wave mode defined as

$$
\Gamma_{ \pm}=\{(\omega \mp m \Omega)[\omega \mp(m h-2) \Omega]\}^{1 / 2} .
$$

The outgoing wave solution in the exterior is given by eq.(22) with a transmission amplitude $A_{ \pm}$. We here assume that the exterior plasma corotates with $\Omega$. The interior solution should be continuously matched with the exterior one at the surface, $z=0$. Thus, the transmission coefficients $T_{ \pm}$for each helicity mode are given by the relation

$$
T_{ \pm}=\left|A_{ \pm}\right|^{2}=1-\left|R_{ \pm}\right|^{2}
$$

Our numerical calculation is limited to $m=1$. The lower bound of the frequency for the positive helicity mode is $\sigma_{+}>0$, that is $\omega>\Omega$. On the other hand, that for negative helicity mode is limited by propagation condition $\sigma_{-}>3 \Omega$, that is $\omega>2 \Omega$. In Fig.3, transmission coefficients $T_{ \pm}$for each helicity mode are shown as a function of angular frequency in inertial frame $\omega$. The rotational effect may be important in the millisecond magnetar model. In the calculation, the star is assumed to rotate with a constant angular velocity, $\Omega /(2 \pi)=10^{3} \mathrm{~Hz}$. This value is somewhat too rapid for SGRs, but we can regard it as an extreme one, which may be present at the newly borne phase. The rotational effect becomes small for much smaller value of $\Omega$. For a comparison, we also show the result $T_{0}$ for the non-rotating case $(\Omega=0)$ by solid curves in Fig.3. The difference between positive and negative helicity states is clear near the low frequency limit, i.e. $\omega<$ a few $\times \Omega$. The difference however becomes almost negligible in the high frequency regime, say, $\omega>10 \Omega$. The transmission rates gradually approach unity with increase of the frequency. The rates also increase with magnetic field strength, but $T_{ \pm} \neq 1$ in the low frequency regime even for $10^{15} \mathrm{G}$. A significant fraction of waves suffers from the bounce at the surface, because of $T_{ \pm} \sim 0.1$ for

$\omega<10 \Omega$. This means that the transmission time to the exterior is roughly estimated not by the crossing time $d / v$, but by $d / v \times T_{ \pm}^{-1}$.

\subsection{Energy and angular momentum loss}

We will calculate the rate of energy and angular momentum carried by the Alfvén waves. The terms linear to the perturbation quantities vanish in the time average. Meaningful time- 


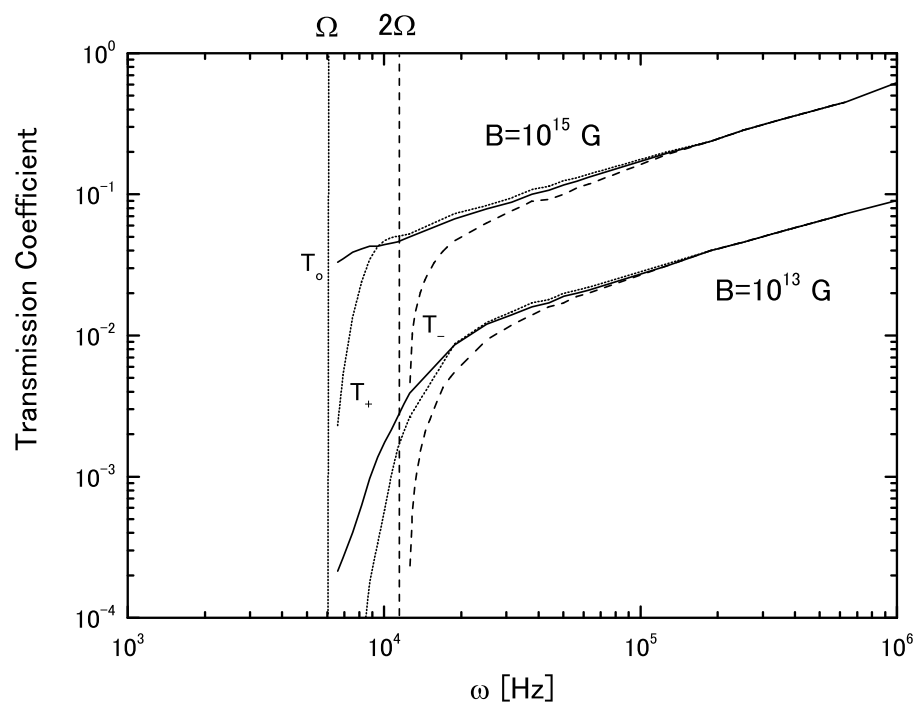

Fig. 3.- Transmission rates as a function of angular frequency for the magnetic field strength $10^{13}$ and $10^{15} \mathrm{G}$. The curves with labels $T_{+}$and $T_{-}$denote results for the positive and negative helicity states. The solid curves with the label $T_{o}$ denote results for the non-rotating star.

averaged values come from square of the perturbation quantities. The time-averaged Poynting flux is therefore given by

$$
\vec{S}=\frac{c}{8 \pi} \Re\left(\delta \vec{E} \times \delta \vec{B}^{*}\right)
$$

where ${ }^{*}$ means complex conjugate and $\Re()$ means taking the real part. We have here used a useful technique in averaging the product of complex quantities with the same harmonics time-dependence as shown in the textbooks, e.g. Jackson (1975). The power $P$ radiated across the surface at $z=0$ is calculated by integrating $S_{z}$ over a circle within $\varpi_{*}$ as

$$
\begin{aligned}
P & =\frac{c}{8 \pi} \int_{0}^{\varpi *} \int_{0}^{2 \pi} \Re\left(\delta E_{\varpi}^{*} \delta B_{\phi}-\delta E_{\phi}^{*} \delta B_{\varpi}\right) \varpi d \varpi d \phi \\
& =\frac{(\omega \mp m \Omega) B_{0}^{2} \varpi_{*}^{2 m} \Re\left(-i \xi_{ \pm}^{*} \frac{d \xi_{ \pm}}{d z}\right)_{z=0} .}{4 m}
\end{aligned}
$$

This is roughly estimated as $P \sim(\delta B)^{2}\left(\pi \varpi_{*}^{2}\right) c$. The power $P_{(R)}$ ejecting to the corotating ambient plasma is evaluated using the boundary condition $(24)$. The power $P_{(S)}$ to the static plasma is also calculated with condition (25). The explicit expressions are

$$
P_{(R)}=\frac{(\omega \mp m \Omega) k_{ \pm} B_{0}^{2} \varpi_{*}^{2 m}\left|\xi_{ \pm}\right|_{z=0}^{2}}{4 m}
$$




$$
P_{(S)}=\frac{(\omega \mp m \Omega) \omega B_{0}^{2} \varpi_{*}^{2 m}\left|\xi_{ \pm}\right|_{z=0}^{2}}{4 m c}
$$

In a similar way, the angular momentum flux per unit time across $z=0$ can be calculated. The transport of the angular momentum results in the torque. The time-averaged torque due to the electromagnetic stresses can be expressed in terms of the surface integral. From the symmetry, non-vanishing angular momentum flux is z-component only, which is expressed as

$$
\begin{aligned}
N_{z} & =-\frac{1}{8 \pi} \int_{0}^{\varpi *} \int_{0}^{2 \pi} \Re\left(\delta B_{\phi}^{*} \delta B_{z}+\delta E_{\phi}^{*} \delta E_{z}\right) \varpi^{2} d \varpi d \phi \\
& =\frac{(\omega \mp m \Omega) \Omega B_{0}^{2} \varpi_{*}^{2 m+2}}{8(m+1) c^{2}} \Re\left(-i \xi_{ \pm}^{*} \frac{d \xi_{ \pm}}{d z}\right)_{z=0} .
\end{aligned}
$$

The angular momentum transfer in eq.(33) comes from the electric part only, since $\delta B_{z}=0$. The value is estimated as $N_{z} \sim\left(\Omega \varpi_{*} / c\right) \times(\delta B)^{2} \varpi_{*}\left(\pi \varpi_{*}^{2}\right)$, and is smaller by an extra factor $\left(\Omega \varpi_{*} / c\right)$ compared with the rough estimate by the magnetic part only. Note that the angular momentum transfer comes from the magnetic term, i.e. $B_{\phi} B_{z}$ for non-relativistic case such as interstellar clouds. Depending on the boundary condition (24) or (25), the angular momentum flux is written as

$$
\begin{aligned}
& N_{z(R)}=\frac{(\omega \mp m \Omega) k_{ \pm} \Omega B_{0}^{2} \varpi_{*}^{2 m+2}\left|\xi_{ \pm}\right|_{z=0}^{2}}{8(m+1) c^{2}}, \\
& N_{z(S)}=\frac{(\omega \mp m \Omega) \omega \Omega B_{0}^{2} \varpi_{*}^{2 m+2}\left|\xi_{ \pm}\right|_{z=0}^{2}}{8(m+1) c^{3}} .
\end{aligned}
$$

It should be noted that the angular momentum flux can be negative by the outgoing waves into the static ambient plasma as shown in eq.(35). This is possible if the condition $(\omega \mp m \Omega) \omega=$ $\sigma_{ \pm} \omega<0$ is satisfied. That is, frequencies, $\sigma$ and $\omega$, are opposite in sign. This condition is the same as the criterion of gravitational radiation reaction instability (Friedman \& Schutz 1978). The oscillation mode counter-rotates when viewed in rotating frame with star, but corotates when viewed in the inertial frame. This provides a mechanism for converting the stellar rotational energy into gravitational radiation. The modes satisfying the condition $(\omega \mp m \Omega) \omega<0$ are very interesting. They carry negative angular momentum. The emission leads to the spin-up of the star.

\section{7. $\quad$ Order of magnitude}

There is a simple relation between the energy flux (30) and angular momentum flux (33) exerted by the Alfvén waves:

$$
N_{z}=\frac{m \Omega \varpi_{*}^{2}}{2(m+1) c^{2}} P .
$$


This holds irrespective of the boundary condition (24) or (25). The total angular momentum loss/gain is related with the total energy radiated by the waves. During the waves emission, the stellar structure may change due to re-arrangement of magnetic fields, and the inertial moment $I$ may be modified. For giant bursts in SGRs, such a possibility is discussed (Ioka 2001; Woods et al. 2001). We do not consider the possibility here. The duration time of the wave emission is denoted by $\tau$. The change in the angular velocity $\Delta \Omega$ is expressed by the total energy $\Delta E_{A}=P \tau$

$$
\frac{\Delta \Omega}{\Omega}=\frac{N_{z} \tau}{I \Omega}=\frac{m \varpi_{*}^{2}}{2(m+1) c^{2} I} \Delta E_{A} \sim 10^{-20}\left(\frac{\varpi_{*}}{10^{4} \mathrm{~cm}}\right)^{2}\left(\frac{\Delta E_{A}}{10^{38} \mathrm{ergs}}\right),
$$

where the total energy by the Alfvén waves is not known, but is used for the typical burst energy in observed in SGRs. There are reports of glitches observed in AXPs(Kaspi et al. 2003), $\Delta \Omega / \Omega \sim 10^{-6}$. Our estimate is too small. Much larger total energy may be expected associated with the giant bursts. Even taking comparable energy observed in X-/ $\gamma$-rays in such events $\Delta E_{A} \sim 10^{44} \mathrm{ergs}$, the change is $\Delta \Omega / \Omega \sim 10^{-14}$, whereas $|\Delta \Omega / \Omega| \sim 10^{-4}$ in the giant burst. One reason of such smallness may come from too much idealized situation, in particular, our model giving $\delta B_{z}=0$ exactly. This introduces a small factor $\left(\Omega \varpi_{*} / c\right) \sim 10^{-6}$ in the angular momentum loss $N_{z}$. Moreover, larger size $\varpi_{*}$ should be required also. Otherwise, a wind of particles and MHD waves or interaction between the crustal neutron superfluid and the rest of the neutron star are more efficient processes for the angular momentum transfer (Thompson et al. 2000).

\section{Discussion}

We have studied the propagation of shear-Alfvén waves through the neutron-star crust to the exterior. Using a simplified cylindrical model, some interesting natures are found. The waves with high frequency, $|\omega /(m \Omega)| \gg 1$, always propagate, whereas those with low frequency propagate or non-propagate depending on spatial position. The transmission coefficient, which depends on the helicity state, is explicitly calculated. The ejection rate to the exterior increases with the frequency of the wave and the magnetic field strength.

We now consider the behavior of modes in low frequency regime. The positive helicity modes $\xi_{+}$always propagate as far as $\sigma_{+}>0$, whereas the negative helicity modes $\xi_{-}$become evanescent near the surface for $0<\sigma_{-}<(m+2) \Omega$. If corotating plasma covers the surface, the disturbances do not propagate in the atmosphere. On the other hand, if such plasma does not exist, i.e. static plasma or vacuum case, then the perturbations revive as outward waves in the exterior, although they suffer from some damping through the interior evanescent region. The exponential decay of the amplitude is not so large, for $\sigma_{-} \sim m \Omega$ with large $m$, as shown in section 2.3. The disturbances therefore penetrate into the surface, and eventually escape to infinity.

When the waves with the frequency $0<\sigma_{-}<m \Omega$ are ejected, they carry away negative angular momentum through the surface. In this case, the torque becomes positive and leads to 
spin-up of the star by the outgoing waves. However, the magnitude is not enough to explain the glitches observed in AXPs(Kaspi et al. 2003). The geometrical factor may be important in actual situation. In our model, the spin axis agrees with the direction of wave propagation, and the direction of the perturbations is orthogonal to the spin axis. For magnetic field line mis-aligned with the rotation axis, the direction of the perturbations is perpendicular to the propagation, but not to the spin axis. In this case, $\delta B_{z}$ is induced in general, and the non-vanishing term $\delta B_{\phi} \delta B_{z}$ results in larger torque. See eq.(33) and the discussion. Compressional fast/slow magnetosonic waves are also induced at the same time. The calculations would be much more complicated.

The increase of angular momentum as a result of radiation is very analogous to that in the gravitational radiation reaction instability, i.e. the CFS mechanism (Chandrasekhar 1970; Friedman $\&$ Schutz 1978). The generic criterion of the instability is $\sigma_{ \pm} \omega<0$ for unmagnetized rotating stars. Some fluid oscillation-modes satisfying this condition can grow in secular time scale as a reaction of gravitational radiation. The low frequency mode satisfies the instability criterion. Related with this, there is a suggestive work by Ho \& Lai (2000). They considered the r-mode instability driven by Alfvén wave emission as well as gravitational one. On the dimensional estimate, the transfer rate by Alfvén wave is larger than that by gravitational wave, for highly magnetized star $B \geq 3 \times 10^{12}(T / 10 \mathrm{~ms})^{-3}$ G. They pointed out the Alfvén wave-driven instability as an efficient process. In their treatment, however, the displacement current is neglected, and hence dynamical degree of the electromagnetic fields is eliminated in a sense. Such an approximation may be justified in the deep interior, but no longer near the surface. Our treatment is concentrated on the crust, but not the interior core, whose bulk motion is important to estimate gravitational radiation. Thus global calculation adequate for the emission of gravitational wave and Alfvén wave is required to determine the efficient mechanism.

As for a fundamental problem of the secular instability, the criterion should be clarified by taking into account of strong magnetic fields. For example, the canonical energy relevant to the instability is constructed only for unmagnetized stars (Friedman \& Schutz 1978). In their treatment of the secular instability, the effect of gravitational emission is neglected in dynamical equation, but should be accounted in the energy equation governing the evolution in longer time scale. Our problem differs in this point. The degree of freedom of the electromagnetic fields is accounted in dynamical equation, and hence Alfvén wave emission from surface is inevitably included. There is no mathematically rigorous proof of the secular stability criterion in magnetized stars, so that further study is necessary to conclude unstable growth of the modes coupled with electromagnetic fields.

Finally, we will comment on some problems in applying to more realistic models relevant to magnetars. The rotation of observed magnetars is very slow $\Omega \sim 1 \mathrm{rad} \mathrm{s}^{-1}$. In order to satisfy the condition $0<\sigma_{-}<m \Omega$, a large azimuthal number, say, $m \sim 10^{3}$ is needed. If there are rapidly rotating stars, a moderate value of $m$ may be sufficient. The other important factor is the magnetic configuration. Thompson et al. (2002) considered the magnetosphere threaded by large-scale electric current. The magnetic field structure is twisted, and quite different from our present model. The 
perturbations of such magnetic fields are induced by bursts, and Alfvén waves propagate. However, the detailed treatment seems to be complicated, and highly numerical calculations are needed. We expect that the behavior of short wavelength, say, much smaller than curvature of magnetic field, may be almost the same as given here. The result of the transmission rate calculated in section 2.5 may be relevant. For the long wavelength mode, geometrical effects are important, and further studies are required.

\section{Acknowledgements}

This work was supported in part by the Grant-in-Aid for Scientific Research (No.14047215, No.16029207 and No.16540256) from the Japanese Ministry of Education, Culture, Sports, Science and Technology.

\section{REFERENCES}

Baym, G., Pines, D. 1971, Ann. Phys. 66, 816

Blaes, O., Blandford, R., Goldreich, P., \& Madau, P. 1989, ApJ, 343, 839

Carroll, B. W., Zweibel, E. G., Hansen, C. J., McDermott, P. N, Savedoff, M. P., Thomas, J. H. \& Van Horn, H. M. 1986, ApJ, 305, 767

Chandrasekhar, S. 1961, Hydrodynamic and Hydromagnetic Stability (London: Oxford University Press)

Chandrasekhar, S. 1970, Phys. Rev. Lett. 24, 611

Cheng, B., Epstein, R. I., Guyer, R. A. \& Young, A. C. 1996, Nature, 382, 518

Friedman, J. L. \& Schutz, B. F. 1978, ApJ, 222, 281

Gavriil, F. P., Kaspi, V. M. \& Woods, P. M. 2002, Nature, 419, 142

Gogus, E. et al. 1999, ApJ, 526, L93

Gogus, E., et al. 2000, ApJ, 532, L121

Ho, W. C. G. \& Lai, D. 2000, ApJ, 543, 386

Ibrahim, A. I., Swank, J. H., Parke, W. 2003, ApJ, 584, L17

Ioka, K. 2001, MNRAS, 327, 639

Jackson, J. D. 1975, Classical Electrodynamics (New York: Wiley) 
Kaspi, V. M., Gavriil, F. P., Woods, P. M., Jensen, J. B., Roberts, M. S. E. \& Chakrabarty, D. 2003, ApJ, 588, L93

Kouveliotou, C., et al. 1998, Nature, 393, 235

Levin, Y. \& D'Angelo, C. 2004, astro-ph/0403014

Lyutikov, M., Thompson, C. \& Kulkarni, S. R. 2002, Neutron Stars in Supernova Remnants ASP Conf. Ser., eds P.O. Slane \& B.M. Gaensler (San Francisco: ASP)

Mereghetti, S., \& Stella, L. 1995, ApJ, 442, L17

Shu, F. H. 1992, The Physics of Astrophysics (California: University Science Books)

Thompson, C.,\& Duncan, R. C. 1993, ApJ, 408, 194

Thompson, C., \& Duncan, R. C. 1995, MNRAS, 275, 255

Thompson, C., \& Duncan, R. C. 1996, ApJ, 473, 322

Thompson, C., 2000, The Neutron Star-Black Hole Connection ed. V. Connaughton et al. (Dordrecht:Reidel)

Thompson, C., Duncan, R. C., Woods, P. M., Kouveliotou, C., Finger M. H., \& van Paradijs, J. 2000, ApJ, 543, 340

Thompson C., Lyutikov, M., \& Kulkarni, S. R. 2002, ApJ, 574, 332

Woods, P. M., Kouveliotou, C., Gogus, E., Finger M. H., Swank, J., Smith, D. A, Hurley, K., \& Thompson, C. 2001, ApJ, 552, 748

Woods, P.M. 2003, High Energy Studies of Supernova Remnants and Neutron Stars COSPAR 2002 (astro-ph/0304372) 